\title{
Chronic Myeloproliferative Disorders: The Role of Tyrosine Kinases in Pathogenesis, Diagnosis and Therapy
}

\author{
Donald Macdonald ${ }^{a}$ Nicholas C. Cross ${ }^{b}$

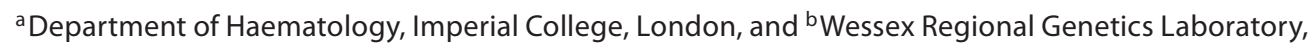 \\ University of Southampton, Salisbury Hospital, Salisbury, UK
}

\section{Key Words}

Chronic myeloproliferative disorders • Leukaemia •

Tyrosine kinase - JAK2 - EMS • Polycythemia - FGFR1 • PDGFR

\begin{abstract}
The term chronic myeloproliferative disorders was originally used by Damashek to describe the link amongst a group of acquired blood diseases. Recent molecular genetic analysis has provided a scientific basis for this observation. Underlying myeloproliferative disorders are acquired abnormalities of tyrosine kinase genes. These may be chromosomal translocations resulting in the creation of a fusion kinase gene, examples of which include ABL, FGFR, and PDGFR as seen in disorders $C M L$, 8p11 myeloproliferative syndrome, atypical CML and chronic eosinophilic leukaemia. The second group of tyrosine kinase abnormalities are point mutations in JAK2, a cytosolic TK. This abnormality is seen in $30-97 \%$ of cases of MPD with the phenotype PV, ET or CIMF.
\end{abstract}

Copyright $\odot 2007$ S. Karger AG, Basel

\section{Introduction}

The study of chronic myeloproliferative disorders (CMPD), a relatively rare group of malignant diseases involving the bone marrow and peripheralblood, has played a significant role in developing some of the key concepts that underlie our understanding of cancer biology. Notable milestones in chronic myeloid leukaemia (CML) include: the demonstration of clonality in a haemopoietic tumour by the use of G6PD isoenzymes, establishing the genetic basis of cancer by the identification of the Philadelphia chromosome, the cloning of a leukaemia-specific fusion gene involving $B C R$ and $A B L$ and finally the advent of molecular targeted therapy using imatinib, an agent designed to inhibit the kinase activity of the $A B L$ fusion gene. Recently there has been substantial progress in our understanding of the biology of other CMPD, so it is an opportune moment to review these developments. In particular, this review considers unifying aspects of molecular pathogenesis of CMPD, new approaches to diagnosis and treatment and speculations as to how recent developments may permit a new disease classification system incorporating molecular genetics.

\section{Chronic Myeloproliferative Disorders}

A link amongst the myeloproliferative disorders was originally proposed in 1951 by Damashek, who recognised that all were characterised by proliferation of one or more myeloid lineages with relatively normal and effective maturation plus the common finding of hepatosplenomegaly and a variable predisposition to transform to acute leukaemia or terminate in bone marrow fibrosis [1]. In 2001 the WHO published a classification of Tu-

\section{KARGER \\ Fax +4161306 1234 \\ E-Mail karger@karger.ch}

www.karger.com
(C) 2007 S. Karger AG, Basel

$1015-2008 / 07 / 0742-0081 \$ 23.50 / 0$

Accessible online at:

www.karger.com/pat
Donald Macdonald

Room 1L05, Charing Cross Hospital

Fulham Palace Road

London W6 8RF (UK)

Tel. +44 208846 7122, Fax +44208846 7111, E-Mail d.h.macdonald@imperial.ac.uk 
Table 1. Myeloproliferative disorders: the current WHO classification and possible future classification incorporating molecular genetics (adapted from Tefferi and Gilliland [29])

WHO classification of tumours of haematopoietic and lymphoid tissues

\begin{tabular}{ll}
\hline Chronic myeloproliferative diseases & Myelodysplastic/myeloproliferative diseases \\
\hline Chronic myelogenous leukaemia (CML) & Chronic myelomonocytic leukaemia (CMML) \\
$\begin{array}{l}\text { Chronic neutrophilic leukaemia } \\
\text { Chronic eosinophilic leukaemia/ } \\
\text { hypereosinophilic syndrome (CEL/HES) }\end{array}$ & $\begin{array}{l}\text { Atypical chronic myeloid leukaemia } \\
\text { Juvenile myelomonocytic leukaemia }\end{array}$ \\
Polycythemia vera (PV) & Myelodysplastic/myeloproliferative disease unclassifiable \\
Chronic idiopathic myelofibrosis (IMF) & \\
Essential thrombocythaemia (ET) & \\
Chronic myeloproliferative disease unclassifiable & \\
\hline
\end{tabular}

Future classification system for myeloproliferative disorders

\begin{tabular}{ll}
\hline Classical myeloproliferative disorders & Atypical myeloproliferative disorders \\
\hline Molecularly defined & Molecularly defined \\
- CML (BCR-ABL-positive) & - PDGFRA rearranged CEL \\
& - PDGFRB rearranged MPD \\
& - FGFR1 rearranged EMS/SCLL \\
& - KIT mutation systemic mastocytosis \\
\hline
\end{tabular}

Clinico-pathologically assigned

(frequently associated with JAK2 ${ }^{\mathrm{V} 617 \mathrm{~F}}$ )

- Essential thrombocythemia

- Polycythemia vera

- Chronic idiopathic myelofibrosis
Clinico-pathologically assigned

(infrequently associated with JAK2 $\mathrm{V}^{617 \mathrm{~F}}$ )

- Chronic neutrophilic leukaemia

- Chronic basophilic leukaemia

- Chronic myelomonocytic leukaemia mours of the Haematopoietic and Lymphoid Tissues. This recognises both the chronic myeloproliferative diseases (CMPD) and an overlap group termed myelodysplastic/myeloproliferative diseases (MDS/MPD) (table 1) [2]. Remarkably, 24 years after Damashek's original hypothesis, we now have supporting molecular evidence that central to the pathogenesis of many CMPD are acquired abnormalities of protein tyrosine kinase genes.

\section{Normal Haematopoiesis and Tyrosine Kinases}

The chronic phase of CMPD retains similarities to normal haematopoiesis. It is worth briefly reviewing some aspects of tyrosine kinase signalling and their role in normal haematopoiesis. Adult haematopoiesis results in the production each day of 400 billion erythrocytes, leukocytes and platelets. Multipotent haematopoietic stem cells pass through stages of differentiation with the progressive loss of developmental options leading to the production of terminally differentiated mature blood cells. This process is regulated by soluble haematopoietic growth factors such as erythropoietin or G-CSF binding to a ligand-specific cell surface receptor present on a precursor cell. This results in transmission of the mitogenic and growth-promoting signals, via signal-transducing proteins to the nucleus [3]. These pathways are key to understanding the biology of CMPD. Most important are two families of cell surface receptors, both of which transmit their growth-promoting signal via the phosphorylation of tyrosine residues. The receptor protein tyrosine kinases (RPTK) are transmembrane receptors which contain an intact catalytic kinase domain in their cytoplasmic portion. The second group are the haemopoietic growth factor receptors, members of the cytokine receptor superfamily which characteristically lack catalytic domains but function by binding and activating a cytosolic (non-receptor) TK protein termed Janus protein tyrosine kinase (JAKs, named after the Roman god with two faces). JAKs contain a catalytic kinase domain [4]. Thus, tyrosine kinases are critical for haemopoietic development. 
Based on human genome sequencing there are more than 520 protein kinases and 130 protein phosphatases which exert reversible control on protein phosphorylation. Both enzyme categories can be further subdivided based on their catalytic specificity into tyrosine kinases or serine/threonine kinases. Protein tyrosine kinases (PTK) have homology over a region of 300 amino acids defined as the catalytic domain which is responsible for the transfer of the phosphate group of ATP to tyrosine residues that may be on the PTK itself (autophosphorylation) or other substrate proteins (transphosphorylation). When reviewed in 2001 there were more than 90 known PTK in the human genome of which 58 encode a transmembrane receptor (RPTK) grouped into 20 subfamilies, and 32 encode cytosolic non-receptor PTK grouped into 10 subfamilies [5].

Structurally, the RPTK consist of an N-terminal extracellular domain which binds ligand, a transmembrane domain, a cytoplasmic juxtamembrane (JM) domain, which in some members has an autoinhibitory function, and a carboxy-terminal kinase domain. In certain subfamilies, e.g. PDGFR, and FGFR, the kinase domain is interrupted by a kinase insert. Ligand binding typically induces receptor dimerisation and reorientation of the intracytoplasmic portion of the receptor, exposing the activation loop and bringing into proximity the two kinase domains. This sets in chain a series of signalling events, the first step of which is the phosphorylation of tyrosine residues in the catalytic domain which activates the kinase function. In addition, tyrosine residues outside of the kinase domain are then phosphorylated and act as docking sites for further signalling molecules via their $\mathrm{SH} 2$ or other phosphotyrosine-binding domains. A range of downstream signalling pathways are consequently activated to promote cell division, growth and survival.

The haematopoietic growth factor receptors in their unbound form exist as dimers with an inactive JAK bound to the cytoplasmic portion. Ligand binding causes reorientation of the intracy toplasmic portion resulting in transphosphorylation of JAK. Activated JAKs use a more direct pathway to transmit its signal to the nucleus via STAT proteins (signal transducers and activators of transcription), in addition to the activation of other signalling pathways. Inactive STAT is bound, phosphorylated and released. Activated STATs form dimers which move to the nucleus where they directly regulate transcription of key genes. Signal transduction by PTKs is summarised in figure 1 .

It is clear that PTKs are central in cell growth and as such their activity within a cell is tightly regulated. Somatic mutations in this small group of genes can release or impair this regulation and are implicated in the development of many cancers. In CMPD, PTK activity is deregulated either through the generation of fusion genes or the acquisition of point mutations.

\section{Tyrosine Kinase Fusion Genes in MPD}

The most extensively studied of the MPD and possibly of all human neoplasms is CML. This was the first example of a MPD where the underlying molecular basis was demonstrated to be a novel fusion gene. The topic of CML is extensively reviewed elsewhere $[6,7]$.

It was noted that rare patients may present with a CML-like disorder in the absence of a $B C R-A B L$ fusion gene. These syndromes could be indistinguishable from classical CML, or alternatively have an atypical clinical course or unusual morphological features such as monocytosis or prominent eosinophilia. In a minority of such cases, conventional bone marrow cytogenetics revealed two rare but recurring abnormalities, translocations that target $8 \mathrm{p} 11$ and those that target 5q31-33. Extensive molecular studies of these rare translocations identified that these translocations resulted in the fusion of a range of fusion partners to a RPTK: fibroblast growth factor receptor 1 (FGFR1) at chromosome 8p11 and platelet-derived growth factor receptor $\beta$ (PDGFRB) at chromosome 5q31-33 [8,9]. Details of the clinico-pathological features of these disorders are set out below, however it should be noted that correct diagnosis of these relatively rare disorders relies on the techniques of morphology and bone marrow cy togenetics to raise clinical suspicion and so direct appropriate further investigations of the underlying molecular genetics.

\section{FGFR1 and PDGFRB Fusion Genes in Myeloproliferative Disorders}

Although rare, accurate diagnosis is important because of their unusual clinical course and to guide therapy. CMPDs associated with either 8p11 (FGFR1) or 5q31-33 (PDGFRB) translocations have both common and distinguishing features. Common features include bone marrow, peripheral blood and clinical findings similar to those of chronic phase CML. There is a prominent eosinophilia and monocytosis. In the original descriptions of patients with FGFR1 translocations, the terms 8p11 myeloproliferative syndrome (EMS) or stem cell leukaemia lymphoma (SCLL) were used $[8,10]$. Patients 
Fig. 1. a Receptor protein tyrosine kinase: ligand binding leads to dimerisation and reorientation of intracytoplasmic kinase domains. Phosphorylation of tyrosine residues leads to activation of kinase domain, release of juxtamembrane autoinhibition, and creation of phosphotyrosine binding sites for downstream signalling molecules. Examples of RPTK families include PDGFR (platelet-derived growth factor receptor), VEGFR (vascular endothelial growth factor receptor), and FGFR (fibroblast growth factor receptor). b Cytokine receptor superfamily: do not contain a kinase domain but exist bound to JAK a cytosolic TK protein. Ligand binding reorientates the cytoplasmic portion and leads to activation of JAK by tyrosine phosphorylation. STAT is then bound via SH2 docking, phosphorylated and released. Activated STAT forms dimers which translocate to the nucleus and mediate gene transcription. Examples include receptors for erythropoietin; interferon $\alpha$ and $\gamma$; interleukin-3; GM-CSF.
Fig. 2. a Diagram of selected RPTK fusion genes. The intact kinase domain is preserved. In FGFR1 and PDGFR $\beta$ fusions the partner gene contains a self-dimerisation motif, e.g. proline-rich domain or HLH (helix loop helix). In chronic eosinophilic leukaemia there is no dimerisation motif in FIP1L1 and the fusion truncates the autoinhibitory JM domain present in PDGFR $\alpha$. TK = Tyrosine kinase; $\mathrm{KI}=$ kinase insert; $\mathrm{JM}=$ juxtamembrane; $\mathrm{TM}=$ trans-membrane. b Network of published fusion genes recognised in MPD. Tyrosine kinases are shown in blue and partner genes in green.

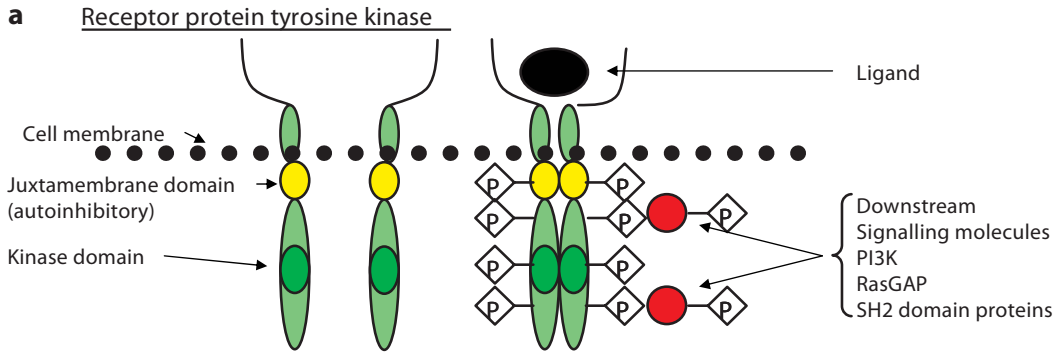

b

Cytokine Receptor Superfamily
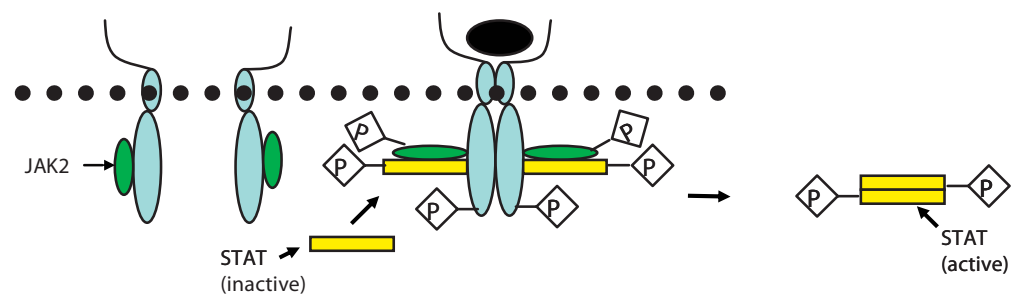

a

ZNF198

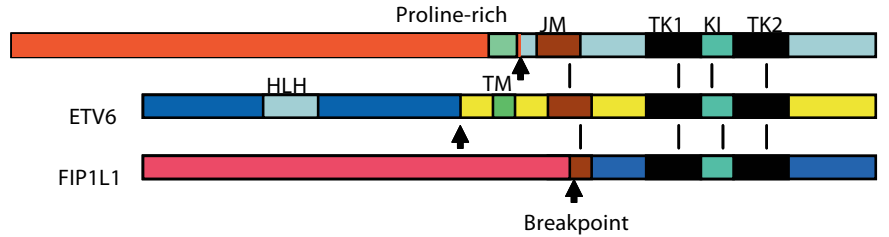

FGFR1

PDGFR $\beta$

PDGFR $\alpha$

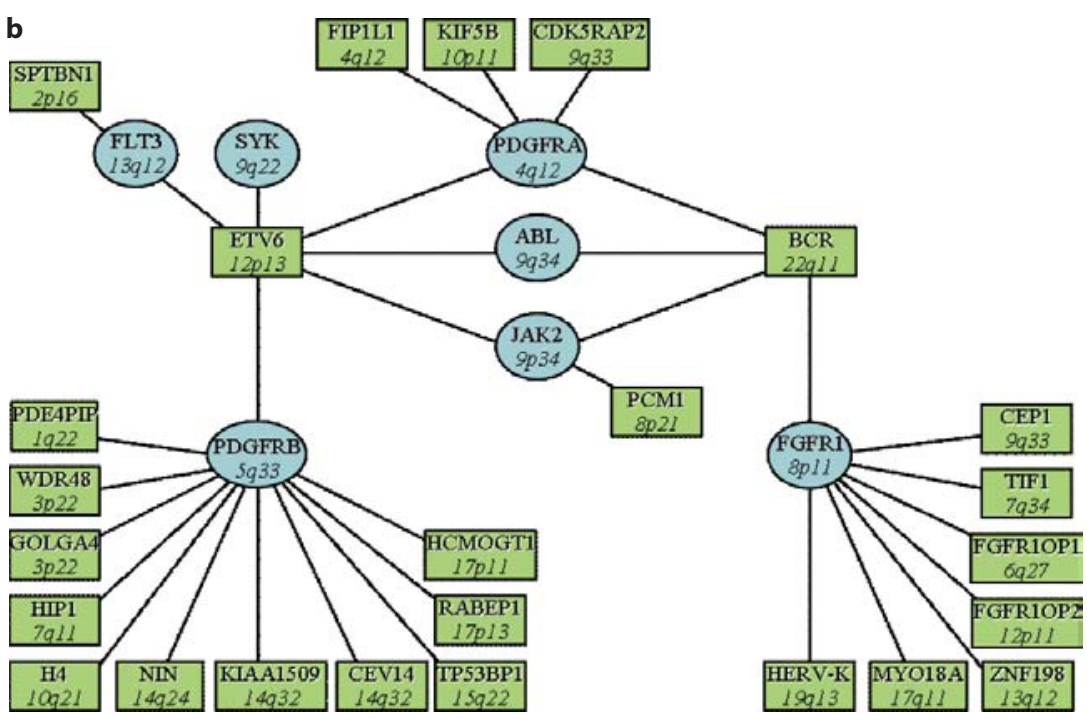


present at a median age of 32 with a slight male preponderance $1.5: 1$. There is a short chronic phase with almost all patients undergoing blastic transformation to acute leukaemia within 1-2 years. Most striking is the high incidence of associated lymphoblastic lymphoma seen in greater than $70 \%$ of cases, suggesting that the target cell for transformation is a common lymphoid/myeloid stem cell. In contrast, PDGFRB translocations present at a median age of 50-60. Blastic transformation occurs in a minority with a latency that ranges from 1 to 12 years, skin involvement may occur, but there is no association with lymphoma. For undetermined reasons there is an extreme male preponderance [9]. Following the initial cloning of FGFR1 and PDGFRB translocations a remarkable number of fusion partners have subsequently been identified (fig. 2) [11].

\section{PDGFRA Fusion Genes in Chronic Eosinophilic Leukaemia}

Chronic eosinophilic leukaemia (CEL) is diagnosed in patients who meet the WHO criteria for hypereosinophilic syndrome (HES), which includes an eosinophilia $>1.5 \times 10^{9} / 1$ persisting for 6 months and who also have evidence of a clonal disorder. Elucidating the molecular pathogenesis of CEL was an example of the so-called bedside to bench approach. Empirically it was recognised that a proportion of patients with idiopathic HES responded to imatinib, the tyrosine kinase inhibitor used in the treatment of CML, suggesting the possible involvement of a PTK. A study to clone an isolated non-recurring chromosomal translocation in one such patient serendipitously revealed the presence of a FIP1L1-PDGFRA fusion gene arising not from the translocation but rather from a cryptic interstitial deletion, del(4)(q12q12), of about 800 $\mathrm{kb}$. Although initial reports suggested that more than half of HES cases were positive for FIP1L1-PDGFRA, more recent data indicates that the true prevalence is approximately $10 \%$. FIP1L1-PDGFRA rearrangements have a strong male preponderance at a ratio of 9:1 $[12,13]$.

\section{How Do Receptor Protein Tyrosine Kinase Fusion Genes Cause Myeloproliferative Disorders?}

The fusion proteins circumvent or are insensitive to the normal tight regulatory inhibition exerted on PTK and are thus constitutively active. The mechanism is best understood for the FGFR1 and PDGFRB fusions, with key observations being that they retain the carboxy-terminal intracytoplasmic kinase domain and contain the N-terminal portion of the partner gene (fig. 2). All the fusion partners described to date contain a dimerisation motif. These result in forced dimerisation and reorientation of the receptor, a process which mimics ligand binding and results in constitutive activation. A further consequence of the translocation is to bring the fusion gene expression under the influence or the regulatory elements which control the $5^{\prime}$ partner gene. This may result in aberrant expression of the RPTK.

Less well understood is the mechanism which leads to CEL in the presence of a FIP1LI-PDGFRA fusion. Whilst the positions of the breakpoints in FIP1L1 are variable, the breakpoint in PDGFRA consistently falls within exon 12. In contrast to the translocations described above, there is no dimerisation motif present in FIP1L1. Recent work has indicated that the conserved PDGFRA breakpoint occurs between the region encoding two tryptophan residues in the JM domain and it is now understood that truncation of the JM domain results in loss of autoinhibition of the RPTK [14].

\section{Diagnosis and Management of MPD Associated with Tyrosine Kinase Fusion Genes}

The diagnosis of CMPD associated with FGFR1 and PDGFRB translocations is based on bone marrow cytogenetics followed by RT-PCR studies to confirm the presence of a fusion gene. In HES, attempts should be made to confirm or exclude the presence of a FIP1L1-PDGFRA fusion gene by RT-PCR and/or FISH [15]. It is important to note that this fusion cannot be detected by conventional cytogenetic analysis.

Normal Pdgfr $\alpha$ and Pdgfr $\beta$ signalling is inhibited by the oral tyrosine kinase inhibitor imatinib. Patients with a PDGFRB fusion gene associated MPD may have a dramatic and sustained response to imatinib [16]. CEL associated with PDGFRA rearrangements responds to imatinib often at low doses in comparison to those used in the management of CML. This may relate to the different pathogenesis, i.e. disruption of an autoinhibitory JM domain as opposed to forced dimerisation or, more likely, because imatinib is much more active against Pdgfr than it is against $\mathrm{Abl}[14,17]$. Normal Fgfrl signalling is not inhibited by imatinib. PKC412 is an alternative oral tyrosine kinase inhibitor that has shown efficacy in a murine model and provided limited short-term disease control prior to stem cell transplant in a patient with FGFR1-as- 
Fig. 3. JAK2 in myeloproliferative disorders. a Sequence trace from 3 individuals who are JAK2 wild-type (normal), heterozygous and homozygous for the $J A K 2^{\mathrm{V} 617 \mathrm{~F}}$ mutation. b In wild-type JAK2 the pseudokinase (JH2) domain forms an intramolecular interaction with the kinase (JH1) domain. The pseudokinase domain inhibits the kinase activity of the JH1 domain. The $J A K 2^{\mathrm{V} 617 \mathrm{~F}}$ mutation lies within the pseudokinase domain and disrupts the intramolecular interaction between $\mathrm{JH} 2$ and JH1. This relieves inhibition of kinase activity. $c$ As a result of the disruption of the autoinhibitory $\mathrm{JH} 2$ domain, JAK2 $2^{\mathrm{V} 617 \mathrm{~F}}$ constitutively activates STAT signalling in the absence of ligand binding by the receptor. a

Normal

Heterozygous

Homozygous

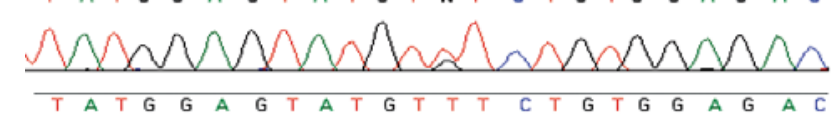

b
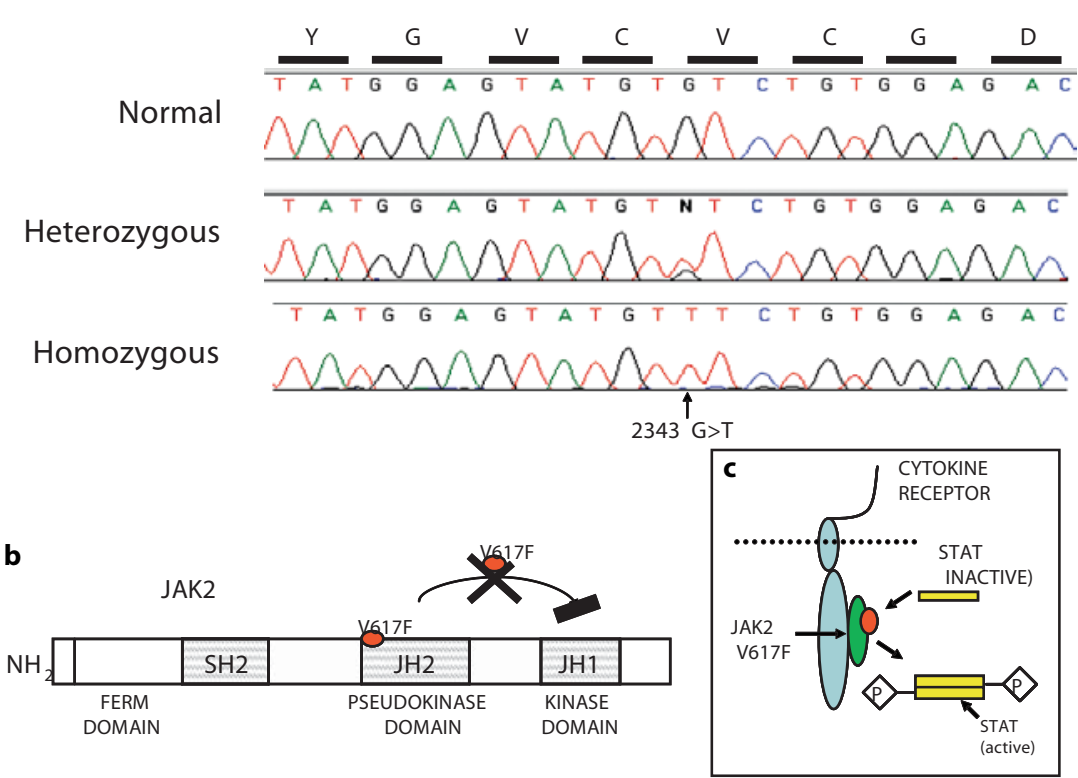

sociated myeloproliferative disease [18]; however, the development of more active compounds is likely to be necessary for effective targeted therapy of EMS/SCLL.

\section{Tyrosine Kinase Gene Mutations in MPD}

The characterisation of RPTK fusion genes provided an insight into the biology of some MPDs. However, it was notable that most were associated with a CML-like phenotype and that no fusion genes were seen in the most common MPDs namely polycythaemia vera (PV), essential thrombocythaemia (ET) and idiopathic myelofibrosis (IMF). Studies of rare inherited MPD families identified mutations which result in elevated thrombopoietin levels in some familial ET and truncation of the erythropoietin receptor in some familial erythrocytosis pedigrees [19]. The implication of EPO receptor signalling and the observation of cytokine-independent growth in vitro of bone marrow colony-forming cells in sporadic PV and ET suggested the possibility of an acquired defect in this pathway. In 2005, five independent research groups identified and confirmed an acquired JAK2 mutation which replaces valine by phenylalanine at position $617\left(J A K 2^{\mathrm{V} 617 \mathrm{~F}}\right)$ in these disorders [20]. The domain structure of JAK2 includes an amino-terminal FERM (band four point one, erzin, radixin, moiesin) domain which mediates binding to the cytokine receptor. At the carboxy-terminal there is both an intact kinase domain JH1, and a non-catalytic pseudokinase domain JH2. The normal JH2 domain, although non-catalytic, plays a role in the regulation of JAK activity. Deletion of the JH2 domain abolishes the response of JAKs to cytokine stimulation, but more pertinent to the pathogenesis of MPD is the observation that deletion of the $\mathrm{JH} 2$ region also results in increased basal activity of JAK2. In the absence of ligand binding there is an intramolecular interaction between the $\mathrm{JH} 1$ and $\mathrm{JH} 2$ domains whereby the JH2 domain exerts an autoinhibitory effect on the catalytic activity of JH1. The JAK2 ${ }^{\mathrm{V} 617 \mathrm{~F}}$ mutation lies within the $\mathrm{JH} 2$ domain and is predicted to interfere with the intramolecular interaction and relieve the autoinhibitory effect exerted by the pseudokinase domain. In cell lines the ectopic expression of mutant JAK2 results in autophosphorylation (in contrast to wild-type JAK2) [21]. This is yet again an example of release of the normal inhibitory constraints on TK activity leading to abnormal cell proliferation (fig. 3).

In different studies the $J A K 2^{\mathrm{V} 617 \mathrm{~F}}$-acquired mutation was found in $65-97 \%$ of cases of PV, and $30-50 \%$ of ET and CIMF. This variable incidence relates mainly to the sensitivity of the assay used. It was also noted that the degree of positivity varied, due to both the size of the af- 
fected clone and whether the mutation was present in the homozygous or heterozygous form. Homozygosity arises from duplication of the mutant allele by mitotic recombination, a process known as acquired isodisomy or uniparental disomy. Although the significance of homozygosity is unclear, it is rare in ET and may be associated with a more severe or pronounced PV or IMF phenotype. Observations such as a higher incidence of complications and a greater requirement for therapy are seen in $J A K 2^{\mathrm{V} 617 \mathrm{~F}_{-}}$ positive compared to negative MPDs [22, 23]. Important questions related to the $J A K 2^{\mathrm{V} 617 \mathrm{~F}}$ mutation currently under investigation are: Why does a single mutation give rise to the diverse phenotypes of PV, ET and IMF? Secondly is the mutation the initiating event for MPD or alternatively a mechanism that provides a proliferative advantage to an already transformed stem cell? Additionally, research is underway to determine if other components of the cytokine receptor/JAK/SAT pathway is mutated in $J A K 2^{\mathrm{V} 617 \mathrm{~F}_{-}}$ negative MPD. Most recently an activating somatic mutation involving the transmembrane domain of the thrombopoietin receptor $M P L^{(\mathrm{W} 515 \mathrm{~L})}$ has been found in $9 \%$ of cases of $J A K 2^{\mathrm{V} 617 \mathrm{~F}}$-negative IMF [24].

Although not considered by the WHO classification as an example of MPD, a review of TK mutations in chronic haematopoietic disorders should note the finding of acquired mutations of the RPTK KIT in systemic mastocytosis. An activating mutation, most commonly $K I T^{\mathrm{D} 816 \mathrm{~V}}$, is found in approximately $80 \%$ of cases [25].

\section{Is Identification of the JAK2 ${ }^{\mathrm{V} 617 \mathrm{~F}}$ Mutation Useful in the Diagnosis of MPD?}

The identification of the JAK2 $2^{\mathrm{V} 617 \mathrm{~F}}$ mutation in almost all cases of PV and approximately half of cases of ET and IMF is undoubtedly a major advance in our understanding of this disease. It has however opened debate on the role of this assay in establishing a diagnosis. It is argued by some that as $J A K 2^{\mathrm{V} 617 \mathrm{~F}}$ is present only in a proportion of cases and since the mutation can also be found at a low frequency in disorders not considered examples of MPD (such as myelodysplastic syndrome 5\% and chronic myelomonocytic leukaemia $3 \%$ ), then mutation detection may play only a minor role in diagnosis. The alternative view is that mutation detection, although clearly not pathognomonic, unambiguously identifies a clonal disorder and will become central in the diagnostic workup of many patients with abnormal blood counts. It is often difficult to distinguish a primary from a reactive elevation in cell counts, and although approaches such as determining clonality using X-linked markers and demonstrating growth factor independent bone marrow colony growth in vitro can be used, they have limitations and are not widely available. An example of diagnostic difficulty is ET, which is currently a diagnosis of exclusion. A model recently proposed includes persistent elevation of the platelet count and identification of $J A K 2^{\mathrm{V} 617 \mathrm{~F}}$ as major diagnostic criteria in ET. It seems certain that future diagnostic schema for MPD will include mutation identification as part of the standard diagnostic investigations [26-28].

\section{Should the Current Classification of MPD Be Revised?}

Disease classification is constantly evolving and increasingly incorporates cytogenetic and molecular genetic information, the paradigm being CML which is diagnosed on the basis of myeloid proliferation and the presence of a $B C R-A B L$ fusion gene. The identification of a range of recurring TK abnormalities has opened up the prospect of a revised classification system. Such an approach has been proposed by Tefferi and Gilliland [29]. Their classification recognises that there are molecularly defined diseases where the mutation is (1) invariably linked to the disorder and (2) can render cells growth factor independent in vitro or cause the disease phenotype in animal models. There are also clinico-pathological associations, an example being $J A K 2^{\mathrm{V} 617 \mathrm{~F}}$ which is associated with a range of disorders including PV, ET and IMF. Their proposal also takes into account conventional clinico-pathological features and on this basis subdivides the diseases into so-called classical myeloproliferative disorders and atypical myeloproliferative disorders (table 1).

\section{Conclusions}

Molecular biology has established a scientific basis for Damashek's original hypothesis regarding MPD. Disruption of the normally tight regulation and inhibition of tyrosine kinase genes either through the creation of fusion genes involving FGFR1, PDGFRA, PDGFRB or ABL with a range of partner genes or alternatively through the acquisition of point mutations such as JAK2 ${ }^{\mathrm{V} 617 \mathrm{~F}}$, or KITT ${ }^{\mathrm{D} 816 \mathrm{~V}}$ plays a central role in these disorders. The recognition of such mutations is increasingly important in clinical diagnosis and seems set to modify the classification of MPD. Finally we are now entering an era where molecularly targeted therapy using oral tyrosine kinase inhibitors will increasingly be available for these disorders. 


\section{References}

$>1$ Dameshek W: Some speculations on the myeloproliferative syndromes. Blood 1951;6: 372-375.

2 Jaffe ES: World Health Organization: Pathology and Genetics of Tumours of Haematopoietic and Lymphoid Tissues. Lyon, IARC Press/Oxford University Press, 2001.

$>3$ Kaushansky K: Lineage-specific hematopoietic growth factors. N Engl J Med 2006;354: 2034-2045.

4 Alberts B: Molecular Biology of the Cell, ed 4. New York, Garland Science, 2002.

$\checkmark 5$ Blume-Jensen P, Hunter T: Oncogenic kinase signalling. Nature 2001;411:355-365.

6 Quintas-Cardama A, Cortes JE: Chronic myeloid leukemia: diagnosis and treatment. Mayo Clin Proc 2006;81:973-988.

$>7$ Melo JV, Deininger MW: Biology of chronic myelogenous leukemia-signaling pathways of initiation and transformation. Hematol Oncol Clin North Am 2004;18:545-568, viiviii.

-8 Macdonald D, Aguiar RC, Mason PJ, Goldman JM, Cross NC: A new myeloproliferative disorder associated with chromosomal translocations involving 8p11: a review. Leukemia 1995;9:1628-1630.

$>9$ Steer EJ, Cross NC: Myeloproliferative disorders with translocations of chromosome 5q31-35: role of the platelet-derived growth factor receptor $\beta$. Acta Haematol 2002;107: 113-122.

10 Inhorn RC, Aster JC, Roach SA, Slapak CA, Soiffer R, Tantravahi R, Stone RM: A syndrome of lymphoblastic lymphoma, eosinophilia, and myeloid hyperplasia/malignancy associated with $\mathrm{t}(8 ; 13)(\mathrm{p} 11 ; \mathrm{q} 11)$ : description of a distinctive clinicopathologic entity. Blood 1995;85:1881-1887.

11 Gotlib J, Cross NC, Gilliland DG: Eosinophilic disorders: molecular pathogenesis, new classification, and modern therapy. Best Pract Res Clin Haematol 2006;19:535-569.

$\checkmark 12$ Cools J, DeAngelo DJ, Gotlib J, Stover EH, Legare RD, Cortes J, Kutok J, Clark J, Galinsky I, Griffin JD, Cross NC, Tefferi A, Malone J, Alam R, Schrier SL, Schmid J, Rose M, Vandenberghe P, Verhoef G, Boogaerts $M$, Wlodarska I, Kantarjian H, Marynen P, Coutre SE, Stone R, Gilliland DG: A tyrosine kinase created by fusion of the PDGFRA and FIP1L1 genes as a therapeutic target of imatinib in idiopathic hypereosinophilic syndrome. N Engl J Med 2003;348:1201-1214.
13 Score J, Curtis C, Waghorn K, Stalder M, Jotterand $\mathrm{M}$, Grand $\mathrm{FH}$, Cross NC: Identification of a novel imatinib responsive KIF5BPDGFRA fusion gene following screening for PDGFRA overexpression in patients with hypereosinophilia. Leukemia 2006;20:827832.

14 Stover EH, Chen J, Folens C, Lee BH, Mentens N, Marynen P, Williams IR, Gilliland DG, Cools J: Activation of FIP1L1-PDGFR $\alpha$ requires disruption of the juxtamembrane domain of PDGFR $\alpha$ and is FIP1L1-independent. Proc Natl Acad Sci USA 2006;103: 8078-8083.

15 Cools J, Stover EH, Gilliland DG: Detection of the FIP1L1-PDGFRA fusion in idiopathic hypereosinophilic syndrome and chronic eosinophilic leukemia. Methods Mol Med 2006;125:177-187.

16 Apperley JF, Gardembas M, Melo JV, Russell-Jones R, Bain BJ, Baxter EJ, Chase A, Chessells JM, Colombat M, Dearden CE, Dimitrijevic S, Mahon FX, Marin D, Nikolova Z, Olavarria E, Silberman S, Schultheis B, Cross NC, Goldman JM: Response to imatinib mesylate in patients with chronic myeloproliferative diseases with rearrangements of the platelet-derived growth factor receptor $\beta$. N Engl J Med 2002;347:481-487.

17 Pardanani A, Brockman SR, Paternoster SF, Flynn HC, Ketterling RP, Lasho TL, Ho CL, Li CY, Dewald GW, Tefferi A: FIP1L1PDGFRA fusion: prevalence and clinicopathologic correlates in 89 consecutive patients with moderate to severe eosinophilia. Blood 2004;104:3038-3045.

18 Chen J, Deangelo DJ, Kutok JL, Williams IR, Lee BH, Wadleigh M, Duclos N, Cohen S, Adelsperger J, Okabe R, Coburn A, Galinsky I, Huntly B, Cohen PS, Meyer T, Fabbro D, Roesel J, Banerji L, Griffin JD, Xiao S, Fletcher JA, Stone RM, Gilliland DG: PKC412 inhibits the zinc finger 198-fibroblast growth factor receptor 1 fusion tyrosine kinase and is active in treatment of stem cell myeloproliferative disorder. Proc Natl Acad Sci USA 2004;101:14479-14484.
19 Skoda R, Prchal JT: Lessons from familial myeloproliferative disorders. Semin Hematol 2005;42:266-273.

20 Zhao R, Xing S, Li Z, Fu X, Li Q, Krantz SB, Zhao ZJ: Identification of an acquired JAK2 mutation in polycythemia vera. J Biol Chem 2005;280:22788-22792.

21 Khwaja A: The role of Janus kinases in haemopoiesis and haematological malignancy. Br J Haematol 2006;134:366-384.

22 Jones AV, Kreil S, Zoi K, Waghorn K, Curtis C, Zhang L, Score J, Seear R, Chase AJ, Grand FH, White H, Zoi C, Loukopoulos D, Terpos E, Vervessou EC, Schultheis B, Emig M, Ernst T, Lengfelder E, Hehlmann R, Hochhaus A, Oscier D, Silver RT, Reiter A, Cross NC: Widespread occurrence of the JAK2 V617F mutation in chronic myeloproliferative disorders. Blood 2005;106:2162-2168.

23 Kralovics R, Passamonti F, Buser AS, Teo SS, Tiedt R, Passweg JR, Tichelli A, Cazzola M, Skoda RC: A gain-of-function mutation of JAK2 in myeloproliferative disorders. N Engl J Med 2005;352:1779-1790.

24 Pikman Y, Lee BH, Mercher T, McDowell E, Ebert BL, Gozo M, Cuker A, Wernig G, Moore S, Galinsky I, Deangelo DJ, Clark JJ, Lee SJ, Golub TR, Wadleigh M, Gilliland DG, Levine RL: MPLW515L is a novel somatic activating mutation in myelofibrosis with myeloid metaplasia. PLoS Med 2006;3:e270.

25 Valent P, Akin C, Sperr WR, Mayerhofer M, Fodinger M, Fritsche-Polanz R, Sotlar K, Escribano L, Arock M, Horny HP, Metcalfe DD: Mastocytosis: pathology, genetics, and current options for therapy. Leuk Lymphoma 2005;46:35-48.

26 Harrison CN, Green AR: Essential thrombocythaemia. Best Pract Res Clin Haematol 2006;19:439-453.

27 McMullin MF, Bareford D, Campbell P, Green AR, Harrison C, Hunt B, Oscier D, Polkey MI, Reilly JT, Rosenthal E, Ryan K, Pearson TC, Wilkins B: Guidelines for the diagnosis, investigation and management of polycythaemia/erythrocytosis. Br J Haematol 2005;130:174-195.

$\checkmark 28$ Tefferi A: The diagnosis of polycythemia vera: new tests and old dictums. Best Pract Res Clin Haematol 2006;19:455-469.

29 Tefferi A, Gilliland G: Classification of chronic myeloid disorders: from Dameshek towards a semi-molecular system. Best Pract Res Clin Haematol 2006;19:365-385. 